\title{
Effect of anticholinergic medications on the risk of dementia: a Protocol of systematic review and meta-analysis
}

\section{Elham Maraghi}

Ahvaz Jondishapour University of Medical Sciences Faculty of Public Health

\section{Ali Reza Safarpour}

Shiraz Medical School: Shiraz University of Medical Sciences

\section{Maryam Hajmohammadi}

Ahvaz Jondishapour University of Medical Sciences Faculty of Public Health

\section{Ali Akbar Oroojan}

Medical University of Dezful

\section{Amal Saki Malehi}

Ahvaz Jondishapour University of Medical Sciences Faculty of Public Health

Hadis Ashrafizadeh ( $\sim$ Ashrafizadeh.h1993@gmail.com )

Ahvaz Jondishapour University of Medical Sciences Faculty of Nursing and Midwifery

https://orcid.org/0000-0002-9369-5854

\section{Protocol}

Keywords: Anticholinergic Drug, Cholinergic Antagonist, Dementia, Alzheimer, Systematic Review, Metaanalysis

Posted Date: March 5th, 2021

DOl: https://doi.org/10.21203/rs.3.rs-271086/v1

License: (c) (1) This work is licensed under a Creative Commons Attribution 4.0 International License. Read Full License 


\section{Abstract}

Introduction: The most kind of dementia is Alzheimer's disease. Alzheimer's is a type of degenerative cerebrovascular disorder which exacerbates over time. Meanwhile, anticholinergic drugs can potentially modify the risk factors. As different studies have achieved different results and the clinical findings of these interventions have not been conclusive, the objective of this research will be to evaluate the effect of anticholinergic drugs on the risk of dementia.

Materials\& Methods: This systematic review and meta-analysis with no language limitation between 1988.12.15 and 2020.12.15 will search WoS, EMBASE, MEDLINE/PubMed, Scopus, ProQuest electronic databases and grey literature. Our search strategy with suitability criteria evaluating the effect of anticholinergic drugs on the risk of dementia. Two authors will independently implement the selection phases, data extraction, and quality assessment. Any discrepancies between reviewers will be resolved by consensus; otherwise, the viewpoint of a third specialist person will be applied. The reviewers will evaluate the risk of bias using NOS. We will conduct a meta-analysis with Random Effect Model or Fixed Effect Model according to severity of methodological heterogeneity. The results will be presented via the forest plot for the composition of final studies' data, to demonstrate the separated and combined frequency and their corresponding $95 \% \mathrm{Cls}$ and summary tables and narrative summaries. Using $\mathrm{I}^{2}$ statistics and the Q-statistic test, the statistical heterogeneity will be evaluated. For evaluating the possible reporting bias, the Funnel plot will be used; also Begg's and Egger's tests will be applied for significant results of the publication bias, and the Fill \& Trim method for reformed publication bias.

Ethical and dissemination: The systematic review findings will be published in a peer-reviewed journal and will also be presented at national/international academic and clinical conferences.

PROSPRO Registration: CRD42020219612

\section{Strengths And Limitations}

- This systematic review and meta-analysis will carefully evaluate the association between Anticholinergic drug use and incident dementia in older adults.

- No language restriction will be applied.

- The included studies' information will be reviewed by three biostatistician.

- Risk assessment will be done by two commonly used tools.

- Imprecision in the pooled effect estimate due to small sample sizes in some studies should be considered as a limitation.

\section{Introduction}

In the last years, the growing rate of the world's elderly population (1.9\%) has significantly exceeded the ascending rate of the world's whole population (1.2\%). It is expected that until 2020 , the population of 
individuals of over 60 years old will reach 2 billion (1) and that of people over 65 years old reach 1.5 billion; in 2030-2050 period, the aging population will increase 3.5 times faster than the total population growth ( $2.8 \%$ vs. $0.8 \%$, respectively). Further, with the rising number of elderly people in the world, the number of people with dementia is expected to triple from 50 million by 2030 to 152 million by 2050(2). Upon the increase in the number of ageing population worldwide, debilitating diseases have significantly increased (3).

One of the most common disorders observed in the elderlies is the cognitive disorder dementia which is associated with severe and progressive disability. $(4,5)$. The most common kind of dementia is senile dementia or Alzheimer's disease (AD)(6). It is a type of degenerative cerebrovascular disorder which aggravates over time. It is believed that Alzheimer's disease inception occurs $\geq 20$ years before the onset of clinical symptoms. (7). The number of prevalent dementia cases between 1990 and 2016 rose by $117 \%$ from 20.2 million in 1990 to 43.8 million. Generally, every three seconds, one person in the world is affected by the disease. Globally, 50 million people live with Alzheimer's disease, and approximately $60 \%$ of them live in low or middle-income countries. In addition, 10 million new cases are diagnosed annually. It has been estimated that the proportion of people aged 60 years and over with Alzheimer's disease, at a certain time, is $5-8 \%$ of the general population (8-12). The exact cause of Alzheimer's disease is still unclear (13). Although the affected people are mostly elderly, the disease is not part of the aging process (10). About $35 \%$ of risk factors of dementia cases are modifiable including high blood pressure, depression, hearing loss, smoking, and diabetes (14). Another modifiable risk factor for dementia is the use of Anticholinergic drugs. Note that long-term use of these drugs increases the risk of dementia. Some anticholinergic drugs work by blocking the effect of acetylcholine on muscarinic receptors within specific organ systems (e.g. gastrointestinal antispasmodics, bladder anti-muscarinics, and anti-Parkinson agents) (15-17).

Due to the importance of the widespread use of anticholinergics, many studies have been conducted to investigate the relationship between the use of anticholinergic medications and the chance of dementia. In 2011, Bhattacharya et al. demonstrated that anticholinergic drugs, though often used in the elderly population, are associated with cognitive disorders and are a significant concern for patients with dementia (18). In 2018, Richardson et al. showed there is a strong relationship between some types of anticholinergic medications and the onset of early signs of dementia in the future (19). In another study in 2018, Richardson et al. showed that the use of anticholinergic drugs is related to dementia in men, but was not observed in women (20). Another study by Coupland et al. in 2019 revealed that concomitant use of more than one potent anticholinergic drug increased the risk of dementia. These results emphasize the need to reduce the use of anticholinergic medications in elderly people (21).

Roger et al. in 2020 showed the use of anticholinergic drugs to control overactive bladder for 3 months increased the risk of dementia by an average of $46 \%$ versus non-use.(22). In addition, the results of a meta-analysis conducted in November 2020 by Nina et al. showed that the use of anticholinergic drugs increases the risk of dementia. However, no causal link can yet be deduced as the studies were associated with a significant risk of bias (22). Nonetheless, researchers plan to increase the comparative 
advantages of the present study to minimize bias. The advantages of the present study include prior enrollment in the PROSPERO along with the most inclusive search done using the thesaurus systems, in comprehensive electronic databases SCOPUS, WOS, MEDLINE via PubMed, EMBASE, Google Scholar, and ProQuest, using the Gray literature including dissertation and conference papers and meeting abstract, key journals and contact with expert persons, Use of high-quality articles and performing subgroup analysis based on the quality of studies, data combining and evaluating the amount, values and finding the causes of possible heterogeneity increase the relative advantages of the present study. Since different studies such as observational and meta-analysis studies have achieved different results and the clinical results of these interventions have not been conclusive, then finding a definitive result to prevent side effects from polypharmacy in the elderly is a research priority worldwide. Based on the above, this study will be performed with the aim of calculating the integrated estimate of dementia risk in patients taking different types of anticholinergic drugs and finding possible sources of heterogeneity and considering randomized clinical trials (RCTs) as acceptable study type.

\section{Aims}

\section{Preliminary Objective}

The preliminary outcome of this research includes assessing the effect of anticholinergic medications on the risk of dementia.

\section{Secondary Objectives}

Estimating the effect of anticholinergic medications on the risk of dementia and Alzheimer's by age group.

Estimating the effect of anticholinergic medications on the risk of dementia and Alzheimer's by gender.

Estimating the effect of anticholinergic medications on the risk of dementia and Alzheimer's by ethnicity.

Estimating the effect of anticholinergic medications on the risk of dementia and Alzheimer's by type of anticholinergic drug.

Estimating the effect of anticholinergic medications on the risk of dementia and Alzheimer's by the different study populations.

Assessing potential heterogeneity in the effect of anticholinergic medications on the risk of dementia and Alzheimer's and finding its possible sources.

\section{Methods}

\section{Design}


This protocol will be presented according to precedent Preferred reporting items for systematic review and meta-analysis protocols (PRISMA-P) 2015 and a meta-analysis of observational studies in epidemiology guidelines (MOOSE) (22).

\section{Suitability Criteria of Primary Studies}

\section{Inclusion and exclusion criteria}

\section{Type of study}

This study will capture prospective and retrospective observational articles (cohort study, case-control, nested case-control) and RCTs that estimate the effect of anticholinergic drugs on the incidence of dementia and Alzheimer's. On the other hand, review article, letter to editor, case series, cross-sectional, clinical trial, short survey, case report and books will not be included. No limitation will be applied on language and sample size for the preliminary studies to be included.

\section{Participant's type}

In observational studies individuals in the case group include all patients diagnosed with dementia or Alzheimer's disease during follow-up after prescription of anticholinergic drugs with any age group, gender, race, and ethnicity. All studies evaluating individuals placed as controls for the case group after matching will be included. In RCTs, studies contained a comparison group that did not have any use of anticholinergic drugs will be considered for inclusion.

\section{Exposure}

The preliminary exposure was to all standardized daily doses of anticholinergic medications determined in years prior to the date of diagnosis of dementia or equivalent date in matched controls (index date).

\section{Search strategy and sources of literature}

\section{Strategy of Search}

This systematic review and meta-analysis with no language limitation between 1988.12.15 and 2020.12.15 will search in the WoS, MEDLINE via PubMed, EMBASE, Scopus, ProQuest, and Google scholar electronic databases. Theses, conference papers, and meeting abstracts will be searched in ISI, Scopus, and ProQuest databases. For finding the synonyms of search components (anticholinergic drug, dementia, Alzheimer's), systems of thesaurus, containing MeSH, Emtree and free text method the experts' opinion, as well as relevant papers and abstracts will be applied. Details on how to search the PubMed database will be provided in the online supplement, Table 1. If we come across studies from other languages such as Portuguese, Chinese, Japanese, etc. we will use the Google translation service and an official translator familiar with that language for more certainty.

\section{Reference lists and Key journals of relevant studies}


A manual search will be performed on the key journals. Based on the analysis of the search results in the databases, the journal election phase will be done. This search will be managed for detecting the journals that have been presented as the greatest storage of sources available on the paper topic, based on the research eligibility criteria. Also, a manual search will be done in the references list of the last papers that would become candidates for quality assessment. These studies will be combined to the last articles' list if we face articles in the former review studies and systematic review studies that have been missed out in the prior search.

\section{Grey Literature}

We will research electronic databases including ProQuest and Scopus to find thesis related to the study subject; also we will contact the experts in the subject area. In addition, electronic databases and information obtained from experts will be used for obtaining conference papers and proceedings. This search will be done manually.

\section{Others}

\section{Contacting expert persons}

In the present study, we will contact experts and ask them to send us any related unpublished studies and dissertations according to the article objectives. Also, we will ask them to introduce conferences relevant to the subject of this research. Further, a manual research of the electronic databases will be done.

\section{Screening and Selection}

Initially, studies retrieved from the search into electronic database will be transferred to EndNote Software version 7 whereby duplicate articles will be removed from the software (EndNote library). Then, during the screening phase, two researchers (HA, MHM) will independently evaluate all of the primary studies based on the title and abstract, and two researchers will check for all studies that match the search strategy to select eligible studies based on the inclusion criteria. The selected articles will be categorized into three groups: related, unrelated, and uncertain. Articles categorized as unrelated by two researchers will be excluded from the study. Afterward, in the selection phase, two researchers (HA, MHM) will independently assess the full texts of the remaining articles. Each researcher will provide a list of selected articles whereby the resulting two lists will be compared. Any discrepancies between researchers will be resolved by consensus and in case no agreement is attained, a third expert person will act as a reviewer (AAO, ARS). The agreement between the two researchers will be evaluated and the result will be reported using the Kappa coefficient and overall agreement.

\section{Risk of bias assessment}

Two reviewers (HA, MHM) will independently assess the risk of bias as well as methodological quality of preliminary studies according to Newcastle Ottawa Scale (NOS) for retrospective and prospective studies (for cross-sectional, nested case control and cohort studies), Cochrane Risk of Bias Tool for Randomized 
Controlled Trials will be used for risk of bias assessment of RCTs and Risk Of Bias In Non-randomized Studies-of Interventions (ROBINS-I) (23-25). NOS scale has eight segments covering parts of selection, comparability, and outcome (25). ROBINS-I scale has seven section of Bias due to confounding, Bias in selection of participants into the study, Bias in classification of interventions, Bias due to deviations from intended interventions, Bias due to missing data, Bias in Measurement of outcomes, Bias in selection of the reported result (23). Cochrane Risk of Bias Tool has seven section of random sequence generation, allocation concealment, selective reporting, and other bias such as: Bias due to problems not covered elsewhere in the table, blinding of participating and personnel, blinding of outcome assessment, incomplete outcome data (26). Any discrepancies between researchers will be resolved by consensus and in case no agreement is attained, a third expert person will act as a reviewer (ARS).

\section{Data extraction}

Two researchers (HA, MHM) will separately do data extraction, using a researcher-made data extraction form. First, an article will be piloted with data extraction form, and then this form will be used for other articles. Each researcher presents the information extracted from an article in the data extraction form where the two forms will be compared. Any discrepancies between two reviewers will be resolved by consensus; otherwise, a third expert person will act as a reviewer (AAO). Then, the agreement between the two researchers will be evaluated. The subsequent data will be elicited from the selected articles: name of first author's, journal name, year of publication, country in which the study was done, design of study, prospective or retrospective design, target population, method of sampling, sample size in the two groups, course of follow-up (length of study), and items relevant to study quality assessment (the score of every segment and the general score of the study quality). The participants' characteristics include age (age groups), gender, ethnicity, smoking, alcohol consumption, history of heart disease, stroke, high blood sugar and hypertension, type of the anticholinergic drugs, and the relationship between the prescription of anticholinergic medications and incidence of dementia or Alzheimer's. If the necessary statistical data are not available in the primary studies, we will contact the corresponding authors and within 10 days, three emails will be sent. We will inform all authors of a given study that their research will be appropriately reported. If we do not receive an answer from the responsible author(s) of a given study after sending three emails, we will remove that study.

\section{Approach to missing and incomplete data}

We will employ one of these studies in the respective combination, if we face duplicate studies. If the data are graphical, we will use Web plot Digitizer software (https://apps.automeris.io/wpd). However, if the data are not graphical, and if it is indispensable to obtain missing data from published articles, writers will be attempted to contact the responsible author via email. In case no response is received within 10 days and their data are related to the primary purposes of the present research, we will exclude the study.

\section{Strategy for data synthesis}


The relationship between the anticholinergic medications and dementia or Alzheimer's risk will be analyzed by pooling odds ratio (ORs) with $95 \%$ confidence interval (Cls) in three models including predominant (TM+MM vs.TT), recursive (MM vs. TM+TT), and homozygote (MM vs.TT) models using STATA Metan module. The $Z$ test will be used to evaluate significant values of the odds ratio (ORs). Heterogeneity between selected articles will be evaluated with a statistical $\mathrm{I}^{2}$ test; Higher $\mathrm{I}^{2}$ values indicate higher levels of heterogeneity across the selected articles (27). For the $Q$ test, the significance level will be $<0.05(28)$.

\section{Statistical analysis}

In the present study, we will use of Hazard ratios and risk ratios as an approximation for the rate ratio and odds ratios (ORs) with a 95\% confidence for dementia associated with cumulative exposure to anticholinergic drugs, adjusted for confounding variables. A Random Effect Model or a Fixed Effect Model based on the methodological similarities of the selected studies will be used appropriately. If heterogeneity is confirmed across the studies, the usual random effects model (DerSimonian and Laird method) is used to integrate the OR index (29). This model simultaneously considers changes between studies and within studies. If meta-analysis is not feasible because of increasing methodological heterogeneity, then based on the results of the studies, only a qualitative narrative discussion will be presented. The $Z$ test will be used to evaluate the significance of the degree of integrated OR and P-Value $<0.05$ will be used as the significance basis of the hypothesis. Further, Forest plots will be drawn for all studies to display the separate and pooled effect size and their corresponding 95\% Cls. Stata V.14.1 (Stata Corp, college station, TX, USA) will be used in the present study.

\section{Evaluation of heterogeneity}

Statistical heterogeneity among primary studies we will be assessed by $\mathrm{I}^{2}$ statistical test, Q-statistic test and corresponding $95 \% \mathrm{Cl}(26)$. We will interpret the $\mathrm{I}^{2}$ coefficient using the following scoring: ( $0 \%$ to $40 \%$ : might not be important; $30 \%$ to $60 \%$ : may represent moderate heterogeneity; $50 \%$ to $90 \%$ : may represent substantial heterogeneity, $75 \%$ to $100 \%$ my represent considerable heterogeneity) (30). For the $Q$ test, the significance level will be $<0.05$ (28).

\section{Subgroup analysis}

We will accordingly use subgroup analysis or meta-regression with restricted maximum likelihood (REML) estimation method for investigating the impact of relevant factors on development of statistical heterogeneity. All studies selected will be divided into different subgroups. In this study, prior subgroup analysis will be done for variables such as age group, gender, ethnicity, alcohol consumption, smoking, dementia or Alzheimer's risk, quality of the study, the drug category, and different doses of the classified use.

\section{Analysis of Sensitivity}


The researchers for sensitivity analysis will implement the one-out remove method. We will attentively check the characteristics of that study if one of the compositions $(\mathrm{K}-1)$ of the articles shows a different result compared to others.

\section{Quality analysis}

For quality analysis, we will assess the relationship between the methodological quality indexes of the primary articles. A reliable, valid, and trustworthy value of the composition of the articles with a minimum acceptable quality will be evaluated if there is remarkable diversity between the finding of the high-quality methodological studies and those of the poor-quality methodological studies.

\section{Assessment of publication bias}

In the study, if adequate numbers of studies more than 10 are included, to assess the publication assess, Funnel plot as well as Begg's and Egger's test will be used. In case the previously mentioned methods show some evidence of publication bias, Fill \& Trim method will be used to correct the effect of publication bias. If the number of studies is fewer than 10 , the publication bias cannot be calculated because of unreliability.

\section{Discussion}

Therefore, the information obtained from this article will be completely reliable. It is expected that our article will have limitations including a high level of heterogeneity regarding the relation of those studies to the times and places, lack of strong population-based studies in most countries and possible methodological bias in the preliminary studies included. It should be remembered that anticholinergic drugs have many different uses and the rate of prescription of these drugs in recent years in people, especially the elderly is increasing. Also, the results of different studies in this field are various, where findings of this study, along with other studies, will help physicians and other health professionals before prescribing these drugs; the elderly, especially those with polypharmacy, should be carefully assessed for the risk of dementia, Alzheimer's or a variety of cognitive disorders. To determine whether the effects of cognitive decline in these drugs are short-term or long-term will be determined in the future by conducting various primary and secondary studies.

\section{Declarations}

\section{Consent for publication}

No patients will be involved in this study

\section{Availability of data and materials}

All data generated or analyzed during this study are included in this published article [and its supplementary information files]. 


\section{Competing interest}

The authors declared no potential conflicts of interest.

\section{Funding}

Ethical approval was granted by student research committee (99s13) and vice chancellor of research of Ahvaz Jundishapur University of Medical science Ethics Committee (IR.AJUMS.REC.1399.173).

\section{Author Contributors}

All authors are involved in the study design, brainstorming and preparation of selected forms, data extraction, and initial drafting. EM, HA, ARS and AAO contributed to the topic refinement as well as formulation of the research question. The preliminary search is done by HA and ARS, and the final search in electronic databases is done by HA and MHM. The screening, selection, quality assessment as well as data extraction process are done by HA, MHM, AAO, as well as ARS as third expert person who will act as a reviewer. Analysis and meta-analysis will be performed by EM, ESM, and ARS. All authors reviewed, edited, and approved the manuscript for submission.

\section{Acknowledgment}

We acknowledge student research committee and vice chancellor of research of Ahvaz Jundishapur University of Medical science for providing the necessary credentials for the present study.

\section{References}

1. Organization WH. 10 facts on ageing and the life course. http://www who int/features/factfiles/ageing/ageing_facts/en/index html. 2014.

2. McNicoll G. World Population Ageing 1950-2050. Population and Development Review. 2002;28(4):814-6.

3. Farhadi A, Noroozian M, Mohammadi F, Foroughan M, Rassouli M, Sadeghmoghadam L, et al. Positive Experiences of Caregiving in Family Caregivers of Older Adults with Dementia: a Content Analysis Study. Iranian South Medical Journal. 2018;21(4):319-34.

4. Craik FI, Salthouse TA. The handbook of aging and cognition: Psychology press; 2011.

5. Dixon RA. New frontiers in cognitive aging: Oxford univ. press; 2004.

6. Colombia mohB. Provincial Guied To Dementia Care in British Colombia 2016.

7. Gaugler J, James B, Johnson T, Marin A, Weuve J. Alzheimer's disease facts and figures. Alzheimers \& dementia. 2019;15(3):321-87.

8. Flaxman AD, Vos DT, Murray CJ. An integrative metaregression framework for descriptive epidemiology: University of Washington Press; 2015. 
9. Brookmeyer R, Corrada MM, Curriero FC, Kawas C. Survival following a diagnosis of Alzheimer disease. Archives of neurology. 2002;59(11):1764-7.

10. organization Wh. Dementia: a global health priority and World Health Organization report. 2019.

11. Iran AAi. 2018.

12. Cummings JL, Cole G. Alzheimer disease. Jama. 2002;287(18):2335-8.

13. Nair M. Nursing management of the patient with Alzheimer's disease. British journal of nursing. 2006;15(5):245.

14. Livingston G, Sommerlad A, Orgeta V, Costafreda SG, Huntley J, Ames D, et al. Dementia prevention, intervention, and care. Lancet (London, England). 2017;390(10113):2673-734.

15. Fox C, Smith T, Maidment I, Chan WY, Bua N, Myint PK, et al. Effect of medications with anticholinergic properties on cognitive function, delirium, physical function and mortality: a systematic review. Age and ageing. 2014;43(5):604-15.

16. Fox C, Richardson K, Maidment ID, Savva GM, Matthews FE, Smithard D, et al. Anticholinergic medication use and cognitive impairment in the older population: the medical research council cognitive function and ageing study. Journal of the American Geriatrics Society. 2011;59(8):1477-83.

17. Gray SL, Anderson ML, Dublin S, Hanlon JT, Hubbard R, Walker R, et al. Cumulative use of strong anticholinergics and incident dementia: a prospective cohort study. JAMA Intern Med. 2015;175(3):401-7.

18. Bhattacharya R, Chatterjee S, Carnahan RM, Aparasu RR. Prevalence and predictors of anticholinergic agents in elderly outpatients with dementia. The American journal of geriatric pharmacotherapy. 2011;9(6):434-41.

19. Richardson K, Fox C, Maidment I, Steel N, Loke YK, Arthur A, et al. Anticholinergic drugs and risk of dementia: case-control study. bmj. 2018;361.

20. Richardson K, Bennett K, Maidment ID, Fox C, Smithard D, Kenny RA. Use of medications with anticholinergic activity and self-reported injurious falls in older community-dwelling adults. Journal of the American Geriatrics Society. 2015;63(8):1561-9.

21. Coupland CA, Hill T, Dening T, Morriss R, Moore M, Hippisley-Cox J. Anticholinergic drug exposure and the risk of dementia: a nested case-control study. JAMA Intern Med. 2019;179(8):1084-93.

22. Dmochowski RR, Thai S, Iglay K, Enemchukwu E, Tee S, Varano S, et al. Increased risk of incident dementia following use of anticholinergic agents: A systematic literature review and meta-analysis. Neurourology and urodynamics. 2020.

23. Sterne JA, Hernán MA, Reeves BC, Savović J, Berkman ND, Viswanathan M, et al. ROBINS-l: a tool for assessing risk of bias in non-randomised studies of interventions. bmj. 2016;355.

24. Higgins J. Cochrane handbook for systematic reviews of interventions. Version 5.1. 0 [updated March 2011]. The Cochrane Collaboration. www cochrane-handbook org. 2011.

25. Stang A. Critical evaluation of the Newcastle-Ottawa scale for the assessment of the quality of nonrandomized studies in meta-analyses. European journal of epidemiology. 2010;25(9):603-5. 
26. Higgins JP, Altman DG, Gøtzsche PC, Jüni P, Moher D, Oxman AD, et al. The Cochrane Collaboration's tool for assessing risk of bias in randomised trials. Bmj. 2011;343:d5928.

27. Nyaga VN, Arbyn M, Aerts M. Metaprop: a Stata command to perform meta-analysis of binomial data. Archives of Public Health. 2014;72(1):39.

28. Higgins JP, Thompson SG, Deeks JJ, Altman DG. Measuring inconsistency in meta-analyses. Bmj. 2003;327(7414):557-60.

29. Jackson D, Bowden J, Baker R. How does the DerSimonian and Laird procedure for random effects meta-analysis compare with its more efficient but harder to compute counterparts? Journal of Statistical Planning and Inference. 2010;140(4):961-70.

30. Deeks JJ, Higgins JP, Altman DG. Analysing data and undertaking meta-analyses. Cochrane handbook for systematic reviews of interventions: Cochrane book series. 2008:243-96.

\section{Table}

Table 1 is available in the Supplementary Files.

\section{Supplementary Files}

This is a list of supplementary files associated with this preprint. Click to download.

- Table.docx

- PRISMAPchecklist.docx 